\title{
Email Attachment
}

National Cancer Institute

\section{Source}

National Cancer Institute. Email Attachment. NCI Thesaurus. Code C81866.

A file that is linked to an e-mail message. 